\title{
6 GENETIC CONTROL OF ANTIBODY PRODUCTION DURING COLLAGEN INDUCED ARTHRITIS DEVELOPIMENT IN HETEROGENEOUS STOCK MICE
}

Michael Förster, ${ }^{1}$ Bruno Raposo, Diana Ekman, ${ }^{1}$ Dorota Klaczkowska, ${ }^{1}$ Marjan Popovic, ${ }^{1}$ Kutty Selva Nandakumar, ${ }^{1}$ Emma Ahlqvist, ${ }^{1,2}$ Rikard Holmdahl ${ }^{1}{ }^{1}$ Section for Medical Inflammation Research, Department of Medical Biochemistry and Biophysics, Karolinska Institutet, Stockholm, Sweden; ${ }^{2}$ Division of Diabetes and Endocrinology, Department of Clinical Sciences, Lund University, Malmö, Sweden

10.1136/annrheumdis-2011-201236.6

Objectives The genetic factors that drive pathogenic autoantibody formation in rheumatoid arthritis (RA) and its experimental models need to be elucidated in order to understand the aetiology of the disease and improve possibilities for therapeutic intervention. For that reason, the authors made a genome wide analysis of quantitative trait loci controlling autoantibody production towards type II collagen (AC2A), anticitrullinated protein antibodies (ACPA) and rheumatoid factors (RF) in the collagen-induced arthritis (CIA) model in mice.

Methods To identify the most important loci controlling autoantibody production, the authors made use of a heterogeneous stock (HS) derived inbred-outbred mouse population, with contribution of eight common inbred mouse strains backcrossed to C57BL/10.O (BQ). Thus, all mice express the arthritis permissive MHC class II gene Aq, which is critical for a type II collagen (CII) immune response. These mice were immunised with CII and subsequently serologically monitored for antibody development by standard ELISA.

Results With this study, the authors have identified numerous loci controlling formation of anti-CII IgG response of different isotypes, antibodies to major collagen epitopes (C1, U1, J1 and F4), to a citrullinated CII peptide (CitC1) and RF. The AC2A, ACPA and RF responses were found to be controlled by distinct genes. AC2A epitope specific response was also controlled by a distinct set of genes, however an important locus was the Immunoglobulin heavy chain ( $\mathrm{IgH})$ locus. Lastly, arthritis 
suppressive antibodies against F4 were uniquely genetically controlled.

Conclusion Here the authors report the genome wide association analysis of the antibody response during CIA development in mice using a HS cohort. AC2A, ACPA and RF antibodies were all controlled by multiple and unique loci. Clearly, our results indicate, that antibody specificity is also determined by non-MHC genes. Surprisingly, only a single locus was found to control AC2A response and clinical disease simultaneously. 